Revista General de Información y Documentación ISSN: 1132-1873

http://dx.doi.org/10.5209/RGID.56562

\title{
La sección Secretaría de los Archivos Históricos de Seminarios, un referente para estudios de Historia de la Educación: el ejemplo del Seminario Metropolitano San Atón de Badajoz
}

\author{
Agustín Vivas Moreno ${ }^{1}$; Guadalupe Pérez Ortiz ${ }^{2}$; Francisco González Lozano ${ }^{3}$
}

Recibido: 28 Septiembre 2016 / Aceptado: 18 de mayo de 2017

Resumen. El Seminario Metropolitano San Atón de Badajoz, como centro formativo de referencia en Extremadura, ha generado a lo largo de su historia un amplísimo patrimonio documental en el campo de la educación y la pedagogía. Prueba de ello la encontramos en su riquísimo archivo. Los investigadores que ahonden en la educación y en los métodos pedagógicos entre los siglos XVII al XX necesariamente volverán su mirada a este interesante archivo. Dentro del cuadro de clasificación del mismo debemos hacer mención especial a la serie documental Secretaría pues es en ella donde encontramos los contenidos más significativos para el estudio de la entidad, así como de los alumnos que han cursado estudios en ella, de los métodos educativos y pedagógicos, etc.

Palabras clave: Archivos eclesiásticos, Archivos de Seminarios, Seminario Metropolitano San Atón, Cuadro de Clasificación, Serie Secretaría, Educación.

\section{[en] The section Secretary of the historical files of seminars, a reference point for studies of history of education: the example of the Metropolitan Seminary San Aton of Badajoz}

\begin{abstract}
The Metropolitan Seminar San Aton Badajoz, as a training centre of reference in Extremadura, has generated throughout its history a wide-ranging documentary heritage in the field of education and pedagogy. Proof of this is found in the wealth of their file. The researchers to ensure in education and in teaching methods between the seventeenth to the twentieth century necessarily your look at this interesting file. Within the classification table of the same we must make a special mention to the documentary series Secretariat because this is where we find the content more meaningful for the study of the entity, as well as students who have studied in it, of the educational methods and pedagogical, etc.
\end{abstract}

Keywords: Ecclesiastical archives, Archives seminars, Seminario Metropolitano San Atón, Classification box, Series secretariat, Education.

1 Facultad de Documentación y Comunicación. Universidad de Extremadura

E-mail: avivas@alcazaba.unex.es

2 Seminario Metropolitano San Atón de Badajoz

E-mail: mgperort@gmail.com

3 Seminario Metropolitano san Atón de Badajoz

E-mail: fglozano@hotmail.com 
Sumario. 1. Introducción. 2. Estado de la cuestión. Aproximación a la historia de la educación en los seminarios. 3. La documentación sobre Educación en los archivos de seminarios: El archivo del Seminario Metropolitano San Atón de Badajoz. 4. Conclusiones. 5. Referencias bibliográficas.

Cómo citar: Vivas Moreno, A., Pérez Ortiz, G., González Lozano, F. (2017) La sección Secretaría de los Archivos Históricos de Seminarios, un referente para estudios de Historia de la Educación: el ejemplo del Seminario Metropolitano San Atón de Badajoz, en Revista General de Información y Documentación 27 (1), 65-85.

\section{Introducción}

La labor educativa realizada por la Iglesia a lo largo de su historia es conocida por todos. El paso de los siglos reclamó la creación de una nueva institución que reglara la formación de aquellos que estaban al frente de las iglesias, los sacerdotes. Fue el Concilio de Trento (Concilium, 1901:628-663) quien ordenó la creación de los seminarios como centros eclesiales educativos destinados a tal fin.

El trabajo que presentamos se focaliza en el Seminario de San Atón, una entidad fundada en la ciudad de Badajoz en mayo de 1664, que ha desarrollado una amplísima labor hasta la actualidad. Su trayectoria educativa ha sido un referente en la cultura extremeña; su prestigiosa influencia formativa, humanística y religiosa ha dejado una huella indeleble en la sociedad a la que sirvió como institución educativa eclesial.

Las posibilidades para este fin son muchas, como los recursos con los que ha contado el Seminario en sus más de tres siglos de historia. De entre todos ellos, nos hemos centrado en uno de los pilares estructurales que constituyen el Seminario, su archivo. Entendemos que en él se van a dar cabida los testimonios escritos que han permitido el desarrollo del centro en su vertiente educativa, además de en otros niveles: económicos, culturales, espirituales, litúrgicos, etc., y que permiten al investigador constatar la relevancia de esta institución.

Los objetivos fundamentales de este trabajo son:

- Dar a conocer el Seminario Metropolitano de San Atón desde la documentación contenida en su archivo (Fuentes Nogales; Pérez Ortiz, 2014).

- Mostrar la importancia que esta entidad tuvo durante los siglos XVII-XIX en el panorama educativo de Extremadura, cuando asume ser la sede del primer Centro de Enseñanza Secundaria en Badajoz y la primera Universidad de Extremadura.

- Dar a conocer los múltiples contenidos educativos y sobre alumnado que aparecen en el cuadro de clasificación del archivo, extrapolable a otros archivos de seminarios, haciendo especial hincapié en la serie SECRETARÍA, dado que en ella se focalizan la mayor cantidad de contenidos sobre el tema.

Para alcanzar los objetivos expuestos se han empleado diversos recursos metodológicos, que han dado lugar a una serie de resultados constatables, que serán expuestos al final del artículo. En primer lugar, el uso de las técnicas archivísticas. 
Dichas técnicas se basaron en el análisis exhaustivo de cada una de las piezas documentales (documentos sueltos) y las unidades archivísticas (expedientes), tanto en sus caracteres internos como externos, haciendo especial hincapié en aquellos documentos que tenían que ver con cuestiones educativas. De la aplicación del método archivístico resulta un fondo documental que presenta una disposición organicista donde cada uno de los documentos sólo tiene sentido en relación con los demás, y preparado para ser consultado por el usuario. En segundo término, el análisis histórico nos permite examinar la documentación desde el punto de vista del investigador interesado en la historia, fundamentalmente en la historia educativa y pedagógica de Extremadura desde el siglo XVII-XIX. Por tanto, ofrecemos contenidos útiles para el historiador y pedagogo que desee hacer un estudio serio de la cultura extremeña en los siglos referidos. Como ya hemos señalado el Seminario san Atón fue el primer centro universitario de Extremadura, la sede del primer instituto de enseñanza secundaria en la ciudad de Badajoz, cuna cultural de miles de niños que elevaron la grandeza de esta tierra mediante la formación académica y promotor insigne de una educación integral debidamente organizada y adecuadamente estructurada.

\section{Estado de la cuestión. Aproximación a la historia de la educación en los seminarios}

El concilio de Trento fue el decimonoveno concilio ecuménico de la Iglesia Católica (1545-1563). A tenor del decreto De Seminariorum erectione et regimine, de 15 de julio de 1563, se dictaminó la creación en todas las diócesis de seminarios.

El nacimiento de una nueva institución orientada a la formación de los clérigos nos conduce directamente a la situación de la Iglesia en un periodo de confusión doctrinal. La teología católica comenzaba a resquebrajarse, por lo que fue necesario un impulso a la formación sacerdotal. Las graves carencias formativas de la Iglesia aquejaban a la misma clerecía. Por tanto, urgía la implantación de seminarios que llevaran a cabo esta misión (Vergara Ciordia, 1996: 499-526). Se presentaba así esta nueva institución como un internado, con enseñanza especial y disciplina particular, siguiendo unos actos de piedad propios de candidatos a órdenes sagradas, bajo la autoridad del obispo, representado en la figura del rector (Rops, 1970: 104).

La producción bibliográfica que recoge la trayectoria más general sobre los seminarios es abundante. El primer estudio sistemático lo encontramos con Juan de Giovanni, Historia de los seminarios clericales, traducida por el maestro Fray Bernardo Agustín de Zamora, por mandato del obispo de Salamanca Don Felipe Beltrán en la imprenta de Francisco Rico, 1778. Pero será el final del siglo XIX y el principio del siglo XX quienes vean la publicación de numerosas obras de referencia para la comprensión y el estudio en profundidad de estas instituciones (Castro Alonso, 1898; Fernández Conde, 1948; Martín Hernández, 1964 y 1973; Sánchez Aliseda, 1942). 
A estas investigaciones generales hemos de sumar otros estudios particulares que analizan épocas posteriores, en concreto entre el concordato de Isabel II y el Concilio Vaticano II; se trata, por tanto, de documentación de necesaria consulta para seguir completando las directrices y trayectorias de los seminarios (Estudios, 1992; Aguirre Prado, 1964; Andrés Martín, 1976; Aznar, 1949; Bartolomé Martínez, 1997; Cárcel Ortí, 1988; Cuenca Toribio, 1973; Faubell, 1993; Turín, 1967; Martín Velasco, 1996; Vergara y Comellas, 2014).

Otros estudios que se centran en cuestiones relativas a los seminarios españoles a partir de estas fechas, que son completados con los análisis específicos sobre la situación de estos centros en los siglos XIX-XX, nos permiten comprender el desarrollo global de estas instituciones desde su nacimiento a épocas actuales (Cuenca Toribio, 1973; Infantes Florido, 1977; Orlandis, 2007; Tineo, 1993; Vázquez Vilanova, 2002).

Nos encontramos, así pues, ante una de las instituciones eclesiales educativas más señeras de la historia. Su misión fundamental fue aclarada desde el principio: la educación de los niños y jóvenes que aspiraban a las órdenes sagradas. Desde esta finalidad educativa podremos comprender, interpretar y valorar la documentación custodiada en sus archivos, método válido para la investigación histórico-pedagógica.

\section{La documentación sobre Educación en los archivos de seminarios: El archivo del Seminario Metropolitano San Atón de Badajoz}

\subsection{Aproximación a la historia del archivo}

La Iglesia, conocedora del rico patrimonio bibliográfico y documental que atesoran sus bibliotecas y archivos, ha demostrado interés en la salvaguarda de los mismos. En relación a los archivos, en un primer momento se pretendía únicamente que fueran elementos que permitieran intemporalizar la doctrina; posteriormente, los archiveros eclesiásticos se encargaron de describir la documentación en ellos conservada, para que los documentos ubicados en archivos eclesiásticos se convirtieran en un elemento más para dar a conocer la labor de la Iglesia a lo largo de los tiempos.

En relación a los seminarios, será a partir del Concilio de Trento (capítulo 18 del Decreto de Reforma) cuando se comiencen a erigir estos centros que surgen para dar una formación integral al clero (Cristiani, 1977: 241-243). Se iniciaba entonces la trayectoria histórica y, por extensión, archivística de una nueva institución.

En el caso de Badajoz se observa la preocupación por la instrucción de los clérigos, pero la escasez de rentas hacía demorar la creación del Seminario. Para su puesta en marcha serán de importancia varios legados, especialmente la obra pía del canónigo Rodrigo Dosma, que cedió sus casas para la erección del centro, como consta en el Libro de Fundación. 
Se instituye el Seminario el 26 de Mayo de 1664, siendo obispo de la diócesis, Jerónimo Rodríguez de Valderas, según refleja el título 27 de las Constituciones (Rubio Merino, 1964: 328).

El archivo, en adelante A.S.M.M.B., se crea a la vez que se instituye el Seminario, suponiendo una ingente producción de documentos. En líneas generales contiene la documentación producida y recibida por la entidad en el trascurso de su actividad educativa, litúrgica, pastoral y la derivada de la conservación y gestión de su patrimonio; así como la de otras instituciones (colegios-seminarios) dependientes de él, todas hoy desaparecidas.

El fondo histórico del archivo queda constituido de la siguiente forma: fondo del Seminario Diocesano de San Atón, fondo del Colegio-Seminario de San Benito (Villanueva de la Serena), fondo del Colegio-Seminario Nuestra Señora de la Coronada (Villafranca de los Barros), fondo de los Seminarios de Elvas y Olivenza, fondo musical y fondo colecciones.

En un primer momento la documentación se encontraba dispuesta en legajos que fueron adaptados en cajas archivadoras para su mejor conservación y custodia. Una vez revisados, la documentación fue cotejada con un catálogo descriptivo previo. En base a ello se confeccionó un cuadro de clasificación, adaptándolo a la Norma ISAD (G). Se presenta este cuadro dividido en seis grandes secciones, de acuerdo a las Constituciones del centro y al Reglamento interno; así como a la propia reglamentación canónica.

\subsection{Análisis educativo a través de los cuadros de clasificación de archivos de seminarios: el ejemplo del A.S.M.M.B.}

El cuadro de clasificación diseñado para el A.S.M.M.B., así como de los fondos dependientes en él custodiados, es una herramienta documental que permite el establecimiento de un instrumento descriptivo de carácter simple que atiende a lo múltiple y heterogéneo de la documentación y a los fondos que sirve; los cuales se dilatan en el tiempo desde el siglo XIV hasta nuestros días, si tenemos en cuanto no solo los fondos históricos de la cuenta sino también su documentación más reciente.

\subsection{GOBIERNO}

\section{CUADRO DE CLASIFICACIÓN}

1.01 Documentación Episcopal

1.01.01 Bulas

1.01.02 Constituciones

1.01.03 Libro Actas de Visita

1.01.04 Libro Decretos Episcopales

1.01.05 Libro Doc. Obispo y Obispado

1.01.06 Libro Fundación Seminario

1.01.07 Libro Inventario Obispado

1.01.08 Reglamentos 
1.02 Documentación Rectorado

1.02.01 Actas Seminario

1.02.02 Libro de Actas Académicas

\subsection{SECRETARÍA}

2.01 Asuntos Académicos

2.01.01 Actas de Exámenes

2.01.02 Becas

2.01.03 Expedientes de Conducta

2.01.04 Listado de Alumnos

2.01.05 Matrículas

2.01.06 Solicitud/Justificantes

2.02 Asuntos Generales

2.02.01 Cédulas Reales

2.02.02 Certificaciones

2.02.03 Correspondencia

2.02.04 Expedientes Personales

2.02.05 Informaciones

2.02.06 Planes de Estudios

\subsection{ADMINISTRACIÓN}

3.01 Administración General

$$
\begin{aligned}
& \text { 3.01.01 Correspondencia } \\
& \text { 3.01.02 Cuentas } \\
& \text { 3.01.03 Obras } \\
& \text { 3.01.04 Pagos } \\
& \text { 3.01.05 Recibos/Justificantes } \\
& \text { 3.01.06 Salarios }
\end{aligned}
$$

3.02 Bienes

$$
\begin{array}{ll}
\text { 3.02.01 } & \text { Censos } \\
\text { 3.02.02 } & \text { Cuentas } \\
\text { 3.02.03 } & \text { Escrituras } \\
\text { 3.02.04 } & \text { Inventario de Bienes } \\
\text { 3.02.05 } & \text { Libro Becerro }
\end{array}
$$

3.03 Obras Pías

$$
\begin{aligned}
& 3.03 .01 \text { Administración } \\
&- \text { Cuentas } \\
&- \text { Inventario de Bienes } \\
&- \text { Libro de Cuentas }
\end{aligned}
$$

3.03.02 Obras Pías

$$
\begin{array}{ll}
\text { - } & \text { Arias de Hoces } \\
\text { - } & \text { Bravo de Laguna } \\
\text { - } & \text { Diego Hernández } \\
\text { - } & \text { Duque de Bejar } \\
\text { - } & \text { Feria }
\end{array}
$$




$\begin{array}{ll}\text { - } & \text { Fonseca } \\ \text { - } & \text { Francisco López Chávez } \\ \text { - } & \text { Francisco Mejías } \\ \text { - } & \text { Josefa Vicente Rino } \\ \text { - } & \text { Marques de Lapilla } \\ \text { - } & \text { Pedro Casas Guerrero } \\ \text { - } & \text { Rodrigo Dosma } \\ \text { - } & \text { Somoza Ribera }\end{array}$

\subsection{FONDOS COLEGIOS-SEMINARIOS DEPENDIENTES}

4.01 Fondo Seminario Menor Elvas-Olivenza
4.01.01 Censos
4.01.02 Correspondencia
4.01.03 Cuentas
4.01.04 Foro de Trigo
4.01.05 Informes

4.02 Fondo Seminario Menor de Nuestra Señora de la Coronada. Villafranca de los Barros 4.02.01 Cuentas 4.02.02 Notas

4.03 Fondo Seminario Menor de san Benito. Villanueva de la Serena
4.03.01. Cuentas
4.03.02 Escrituras
4.03.03 Matrículas
4.03.04 Notas
4.03.05 Solicitudes

\subsection{FONDO MUSICAL}

5.01 Música sacra

5.02 Música profana

\subsection{FONDO COLECCIONES}

6.01. Fotografías

6.02 Monedas

A groso modo, el cuadro de clasificación del A.S.M.M.B., extrapolable a otras entidades similares, se constituye de 6 secciones: gobierno, administración, secretaría, fondos dependientes, archivo musical y colecciones.

- La primera de las secciones, GOBIERNO, contiene la documentación que ha generado el Seminario en su gestión interna, haciendo alusión a los elementos constitutivos más importantes de la entidad.

- La segunda de las secciones, SECRETARÍA, da cabida a la parte más funcional de los seminarios. Como entidades educativas que son, van a establecer una clara distinción entre una secretaría general, que puede ser extrapolada a cualquier otra entidad o empresa, y una secretaria académica, que va a gestionar asuntos específicamente relacionados con la docencia. 
- La tercera de las secciones, ADMINISTRACIÓN, está relacionada con las actividades encaminadas a la gestión de los bienes del Seminario, así como de los colegios dependientes de él.

- La cuarta sección, FONDOS COLEGIOS-SEMINARIOS DEPENDIENTES, da a conocer documentación de los colegios-seminarios que dependieron del de Badajoz y que se crearon en la diócesis para dar formación a niños y jóvenes de poblaciones lejanas a la ciudad de Badajoz, donde se ubicaba el Seminario.

- La quinta sección, FONDO MUSICAL, se compone de partituras de música sacra y profana. Su estudio enmarcará la promoción musical en muchos de los pueblos de la región, puesto que gran parte los directores de corales adquirieron esta formación en el Seminario.

- La sexta sección, FONDO COLECCIONES, queda integrada por dos grandes subsecciones que se dedican a la fotografía y a las monedas.

\subsection{La sección Secretaría de los Archivos Históricos de Seminarios punto de partida para los estudios sobre Historia de la Educación: el ejemplo del Seminario San Atón de Badajoz}

Según el cuadro de clasificación anteriormente expuesto varias son las secciones que conservan documentación relacionada con la educación: gobierno y administración son dos de ellas; aunque sin lugar a dudas es la sección secretaría en la que se recogen la mayor cantidad de documentos relacionados con el campo analizado.

2.01 Asuntos académicos: es sin lugar a duda la subsección más importante de los cuadros de clasificación de archivos de seminarios, dado que acumula la documentación más significativa. En líneas generales da cabida a los contenidos relativos a la matriculación y trayectoria académica de los alumnos, además de todo lo referido a las becas que facilitaban el acceso y la permanencia en el centro. Veamos cada una de las series documentales que componen la subsección:

2.01.01 Actas de exámenes: las actas de exámenes son relaciones de notas de los alumnos que cursan sus estudios en un determinado centro.

Se abre la puerta a un estudio exhaustivo del modo de calificación del profesorado, de las observaciones marginales que apuntaban y que detallan la evolución académica de los alumnos, de las cuestiones en las que algunos alumnos debían incidir para superar las materias, etc.

En el caso concreto del seminario pacense abarca las notas expedidas por el profesorado a sus alumnos entre 1858 y 1930. 


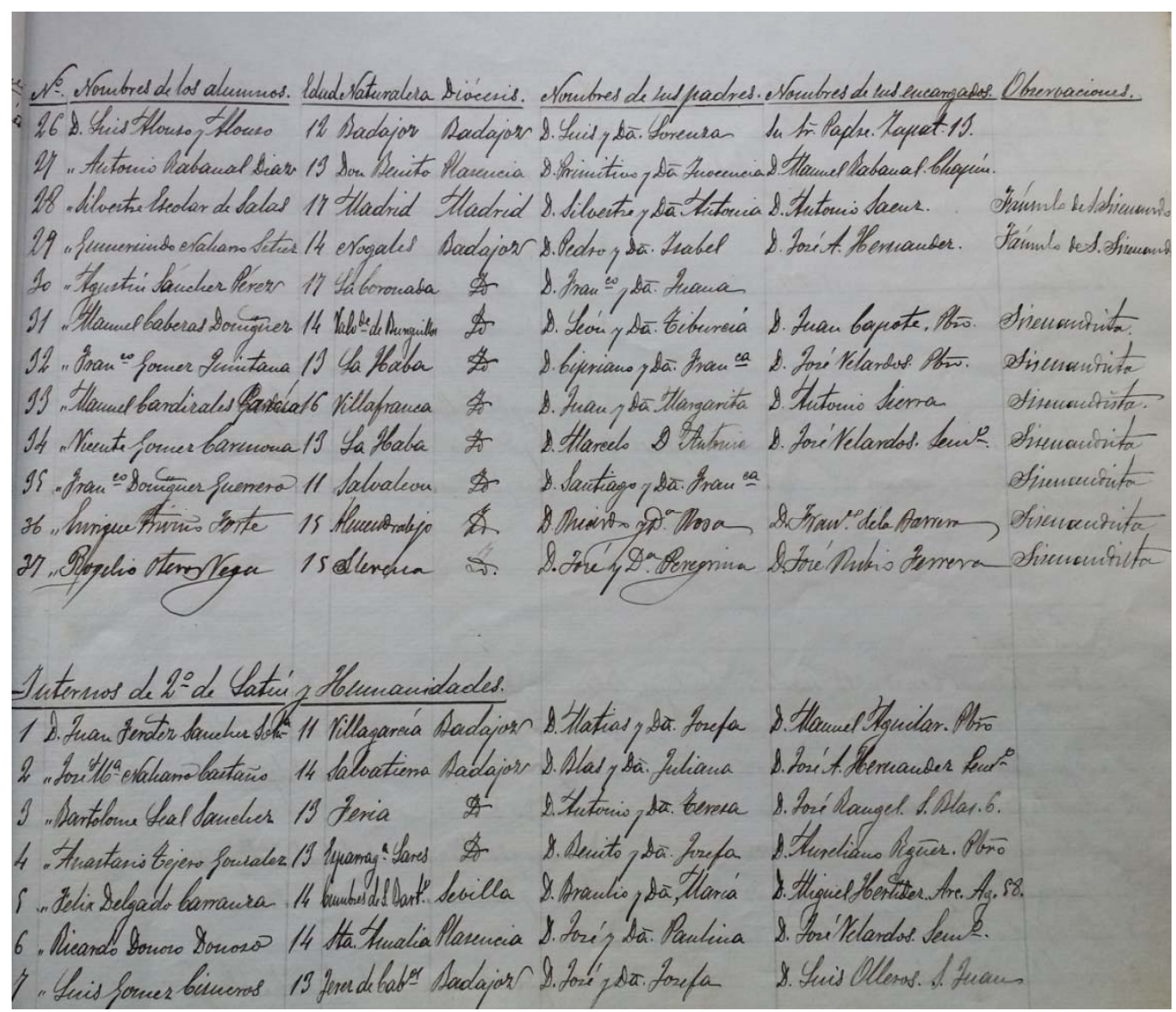

Figura 1. Actas de exámenes

2.01.02 Becas: los seminarios, desde el punto de vista económico, no cerraron sus puertas a los más necesitados. Es más, se podría afirmar, con la información contenida en esta serie, que fueron muchos los que pudieron acceder a estudios mediante el sistema de ayudas instituidas por los obispos.

Este hecho repercutirá positivamente en la disminución del analfabetismo de las sociedades donde se insertan estos centros, porque permitieron que muchos alumnos pudieran acceder a una educación de calidad sin coste alguno.

El expediente de becas quedaba constituido por la solicitud del candidato, primer paso que el seminarista debía emprender para iniciar sus estudios en un seminario. Los alumnos que deseaban ingresar debían enviar al obispo o al rector una súplica o petición informando de los deseos que le llevaban a querer ingresar en este centro. No debemos olvidar que se trata de una entidad donde se iban a formar los futuros sacerdotes y esta cuestión debía ser la base fundamental del documento. En estos documentos se incluye además de los deseos vocacionales, información detallada del alumno y de sus progenitores, así como de su procedencia, lo que nos permitirán trazar interesantes estudios estadísticos; 
contenidos relativos a la pobreza del candidato, solicitando ya desde este momento la posibilidad de optar a beca o exención del pago de matrícula.

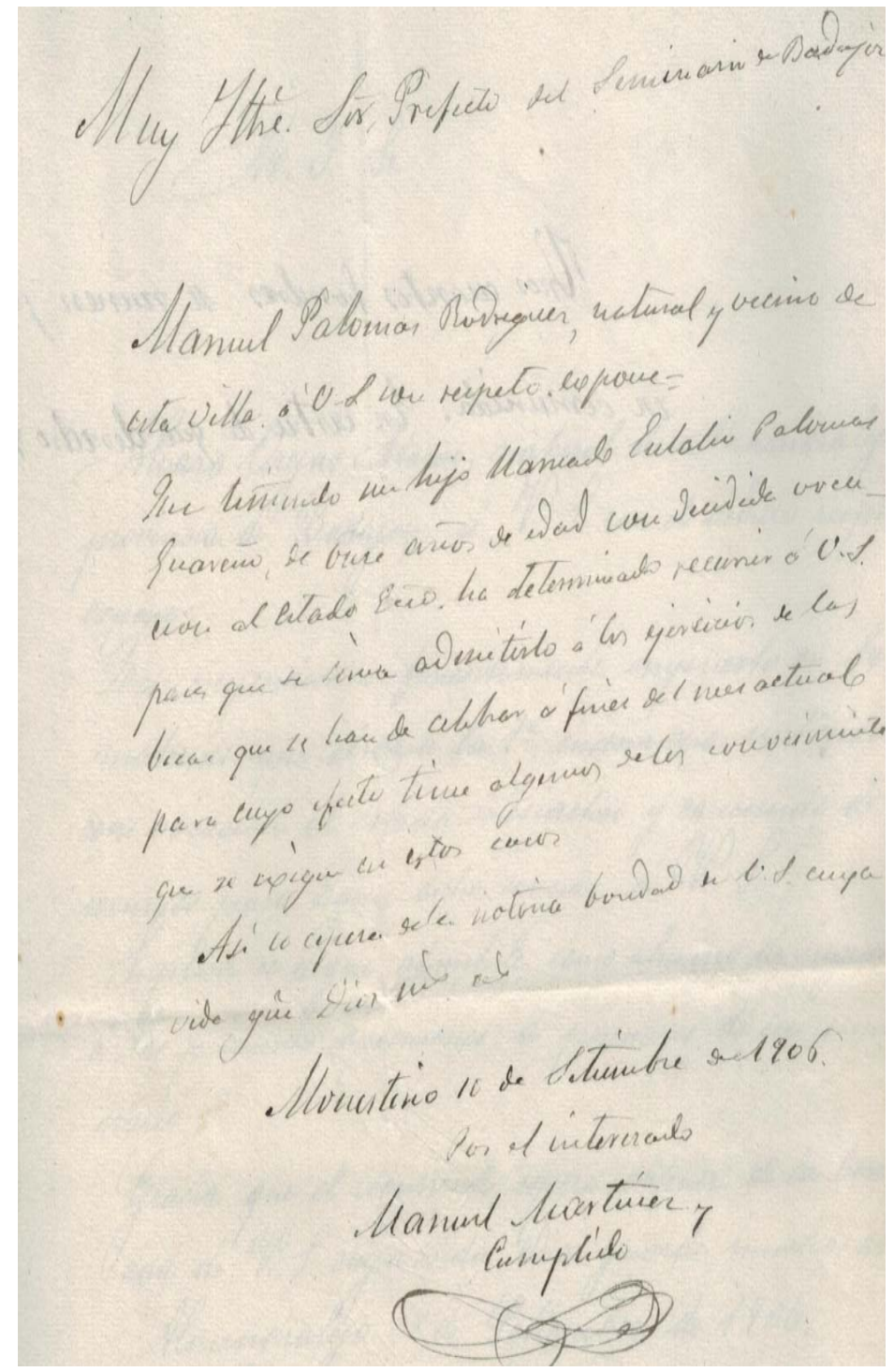

Figura 2. Solicitud para la concesión de beca

En algunos casos este expediente iba acompañado de un documento de carácter civil, expedido por el alcalde de la población de la que provenía el alumno, indicando su condición de pobreza. 
Vivas Moreno, A.; Pérez Ortiz, G.; González Lozano, F. Rev. gen. inf. doc. 27(1) 2016: 65-85

75

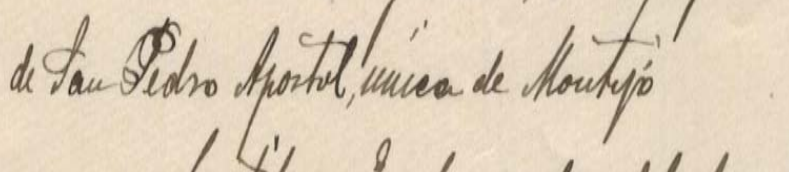

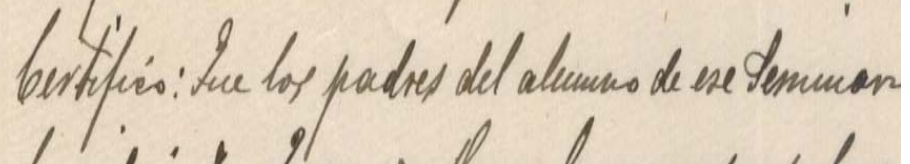

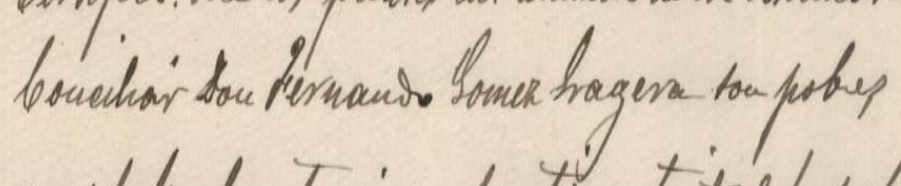

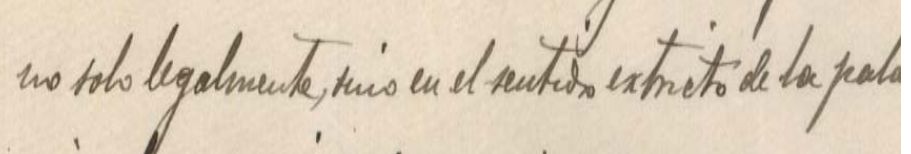

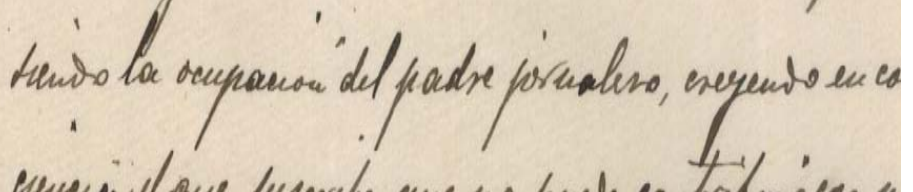
envei il que tusorche que no puede enctribuir coa ne a'la camera de tue hyp'

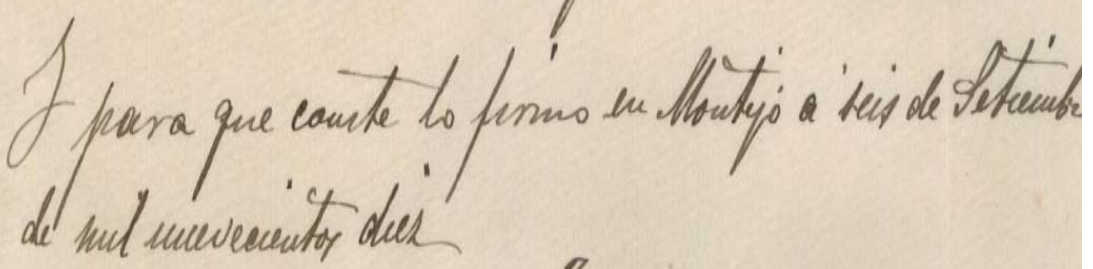

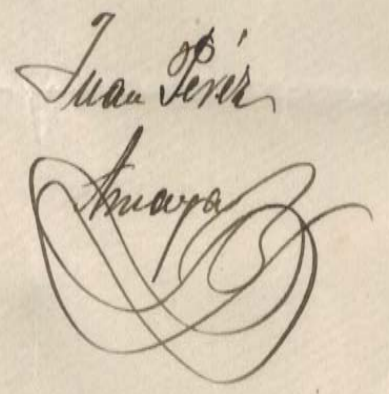

Figura 3. Certificado de pobreza 
Junto a los documentos anteriores se añadía uno final, que en muchos casos no era más que una nota marginal, en la que se indicaba la concesión o no de la gracia solicitada.

2.01.03 Expedientes de conducta: los seminarios concebían la educación como un proceso más amplio que la mera exposición de contenidos que habrían de memorizarse. La dimensión integral de la formación ofertada hacía que en el periodo vacacional se hiciera un seguimiento de los alumnos. Los párrocos rellenan estos expedientes de conducta aportando información muy valiosa sobre el modo de vida de los colegiales. De este modo, aquellos que accedían al seminario venían respaldados por informes que los acreditaban como niños y jóvenes de buena conducta.

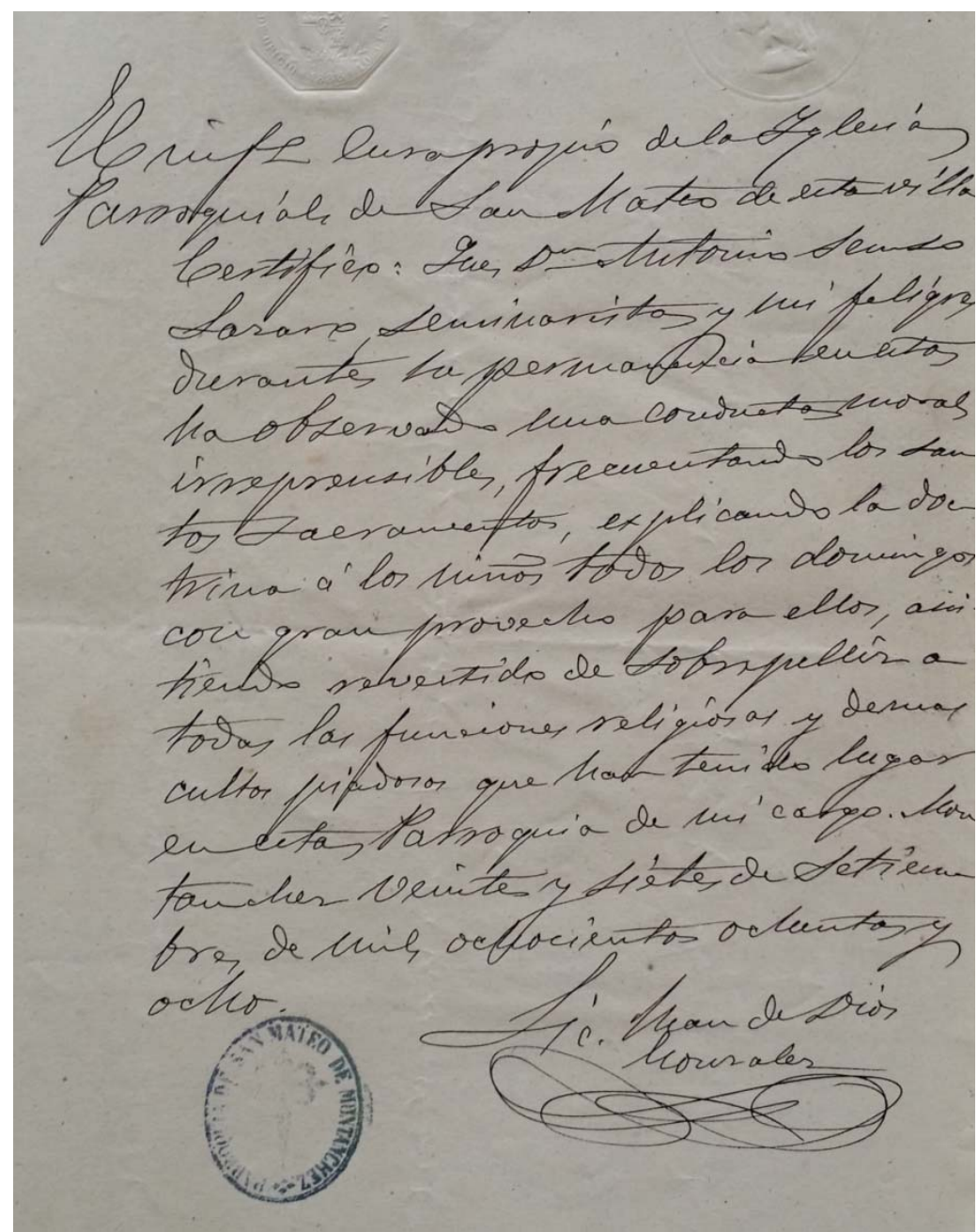

Figura 4. Certificado de buena conducta 
2.01.04 Listado de alumnos: los listados de alumnos no son más que relaciones nominales de los colegiales que pasaron por los seminarios. Sin embargo, para el investigador esta serie documental puede aportar contenidos muy relevantes: número de matrículas, procedencia de los alumnos, edad, nombre de los padres, observaciones de variada índole, especialmente interesantes son las relativas al comportamiento de los alumnos, etc.

El A.S.M.M.B. custodia relaciones nominales de alumnos entre 1853 y 1895, su análisis mostrará la influencia educativa del centro como referente en la región a lo largo del siglo XIX, dado que permite observar el gran número de alumnos que pasaron por sus aulas en este periodo.

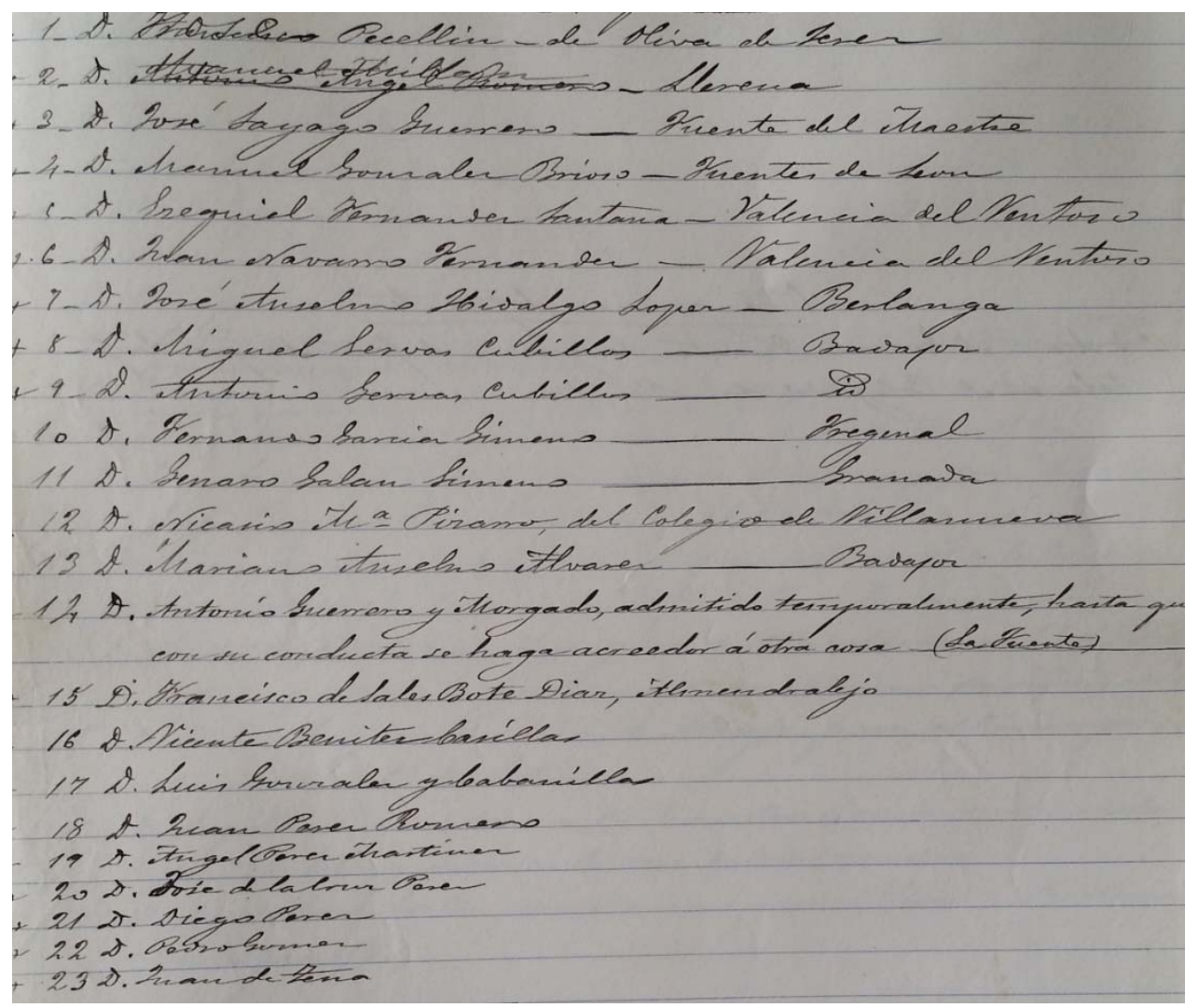

Figura 5. Listados de alumnos

2.01.05 Matrículas: mediante el análisis de matrículas se puede completar la serie anterior. Se abre también la puerta al estudio de casos sobre algún curso o alumno particular, observando la relevancia social que muchos de los alumnos tuvieron a nivel civil.

2.01.06 Solicitud/Justificantes: esta documentación es de sumo interés para completar los estudios individuales o grupales que los investigadores emprendan 
sobre la educación en la provincia de Badajoz desde el siglo XVI, especialmente significativa para los siglos XVII y XVIII.

Da cabida, por una parte, a las solicitudes que los candidatos dirigían al obispo o al rector para la realización de cualquier trámite. Son especialmente significativas las que se solicitan para poder realizar exámenes fuera de plazo o para la salida temporal del seminario por asuntos familiares o personales. Por otra parte, aparecen en esta serie justificantes de variada índole, los más significativos y abundantes son de notas.

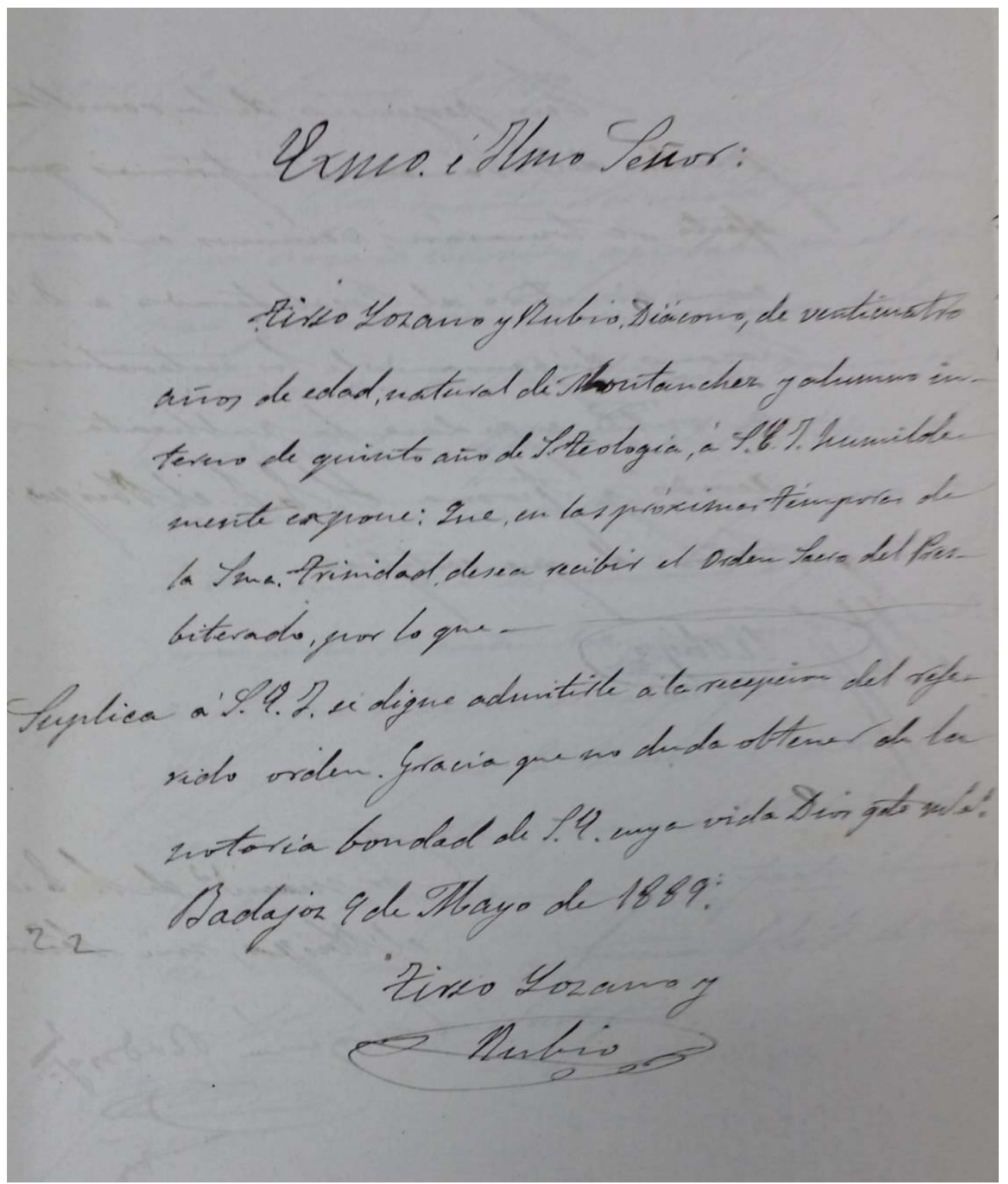

Figura 6. Súplica para realización de examen 
2.02 Asuntos generales: la secretaría general debía publicar los planes de estudio que se impartían en el centro. Además se llevaba detallada cuenta de los certificados que los alumnos solicitaban o aquellos que se enviaban a las universidades a las que estuvo adscrita el Seminario.

2.02.02 Certificaciones: entre 1828 y 1904 el secretario del centro detalla los múltiples certificados expedidos a los alumnos. De ellos se pueden sacar datos muy valiosos para seguir la trayectoria biográfica de los mismos.

2.02.03 Correspondencia: aquí encontramos, fundamentalmente, las solicitudes personales que los alumnos enviaban al obispo para obtener beca o la misma licencia solicitada para presentarse a algún examen. Además se encuentra la correspondencia del obispo al rector y viceversa sobre temas relacionados con el Seminario. También localizamos los nombramientos de catedráticos de los distintos centros y las cartas de relaciones con otros centros académicos de la ciudad o de otras regiones.

2.02.04 Expedientes personales: es sin lugar a dudas una de las series documentales más importantes por su variedad, volumen y cronología de los cuadros de clasificación de archivos de seminarios. De ella podremos obtener un elevado número de datos biográficos referidos al alumnado. En el caso del seminario pacense, llama la atención por su amplitud, 42 cajas de expedientes personales.

Los candidatos para ingresar al seminario debían presentar una documentación previa que sería revisada por el obispo o por el rector. En líneas generales, salvo casos excepcionales, sus expedientes contaban con una solicitud previa del candidato dirigida personalmente al obispo o al rector (existen casos en los que dicha solicitud era firmada por el padre); la aprobación y visto bueno de los últimos responsables eran necesarios para la admisión del candidato. Habrían de presentar también un certificado de bautismo que acreditara la catolicidad del niño. Se incluye un certificado de buena conducta visado por el párroco donde residía habitualmente el candidato. Estos últimos datos nos dan una visión del contacto asiduo que existía entre seminario y parroquia y que ambos iban de la mano en la consecución de los fines educativos y su formación. Igualmente se adjuntaba un certificado médico que acreditara la ausencia de enfermedad contagiosa o infecciosa. En el caso de haber asistido a alguna escuela de gramática o haber cursado enseñanza privada en alguna de las escuelas rurales existentes en la provincia, añadían un certificado de dichos estudios. Esta última documentación ayuda en la elaboración del mapa educativo de la provincia, puesto que sitúa las poblaciones en las que estas escuelas de enseñanza doméstica, privada o escuelas de gramáticas (dependiendo de la época que se estudie), además de proporcionar información sobre los directivos de dichas escuelas. Muchos de los candidatos incluían un certificado de pobreza y una solicitud de beca, lo cual aporta datos relevantes en cuanto a la contribución cultural del seminario a una región eminentemente rural.

En orden a adquirir una visión panorámica aquél que se adentre en el estudio pedagógico de esta institución debe proceder a la consulta de estos expedientes personales. Mediante ellos nos hacemos idea del número de alumnos que pasó por 
las aulas de San Atón, sus procedencias, en algunos casos la situación laboral de sus padres, cuál era su situación académica previa a la entrada al seminario, incluso la situación económica de penuria que nunca impidió al centro ofrecer a la sociedad una educación de alto nivel mediante un sistema de becas sostenidas por el obispo y otros particulares. Así mismo relatan, con todo lujo de detalles, el modo de realizar el examen de grado en Teología que se acordó en el concordato de 1851.

2.02.05 Informaciones: completa la sección anterior y da una visión cronológica previa: 1683-1853. Resulta imprescindible su consulta para hacerse una composición de lugar del alumnado de la época.

2.02.06 Planes de estudio: a partir del concordato de 1851, la titulación ofrecida por la gran mayoría de seminarios españoles se redujo al grado de bachiller en Teología, limitándose la obtención de los grados de licenciatura y doctorado a unos pocos centros repartidos por la geografía española. Se había iniciado una nueva adaptación pedagógica que se llevó a cabo mediante la adaptación al nuevo plan. De esta serie se entresaca el listado completo de asignaturas que se cursaban en las diferentes etapas: Latín y Humanidades, Filosofía y Teología. A estos estudios se les añadían los que seguían la Carrera Menor, un modo más abreviado para acceder al ministerio sacerdotal.

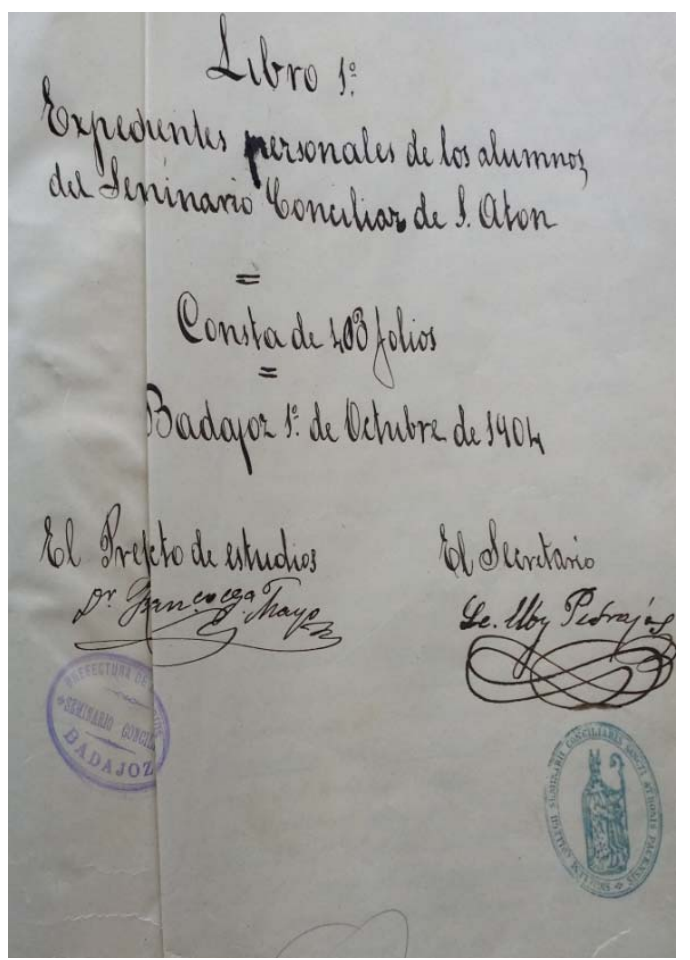

Figura 7. Portada libro de expedientes personales

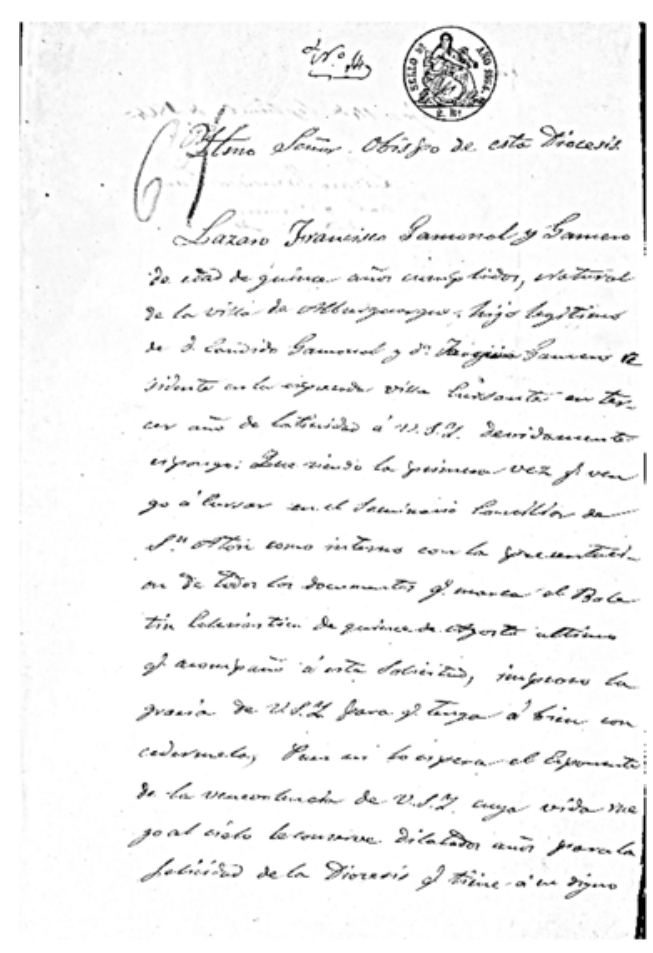

Figura 8. Solicitud de ingreso 


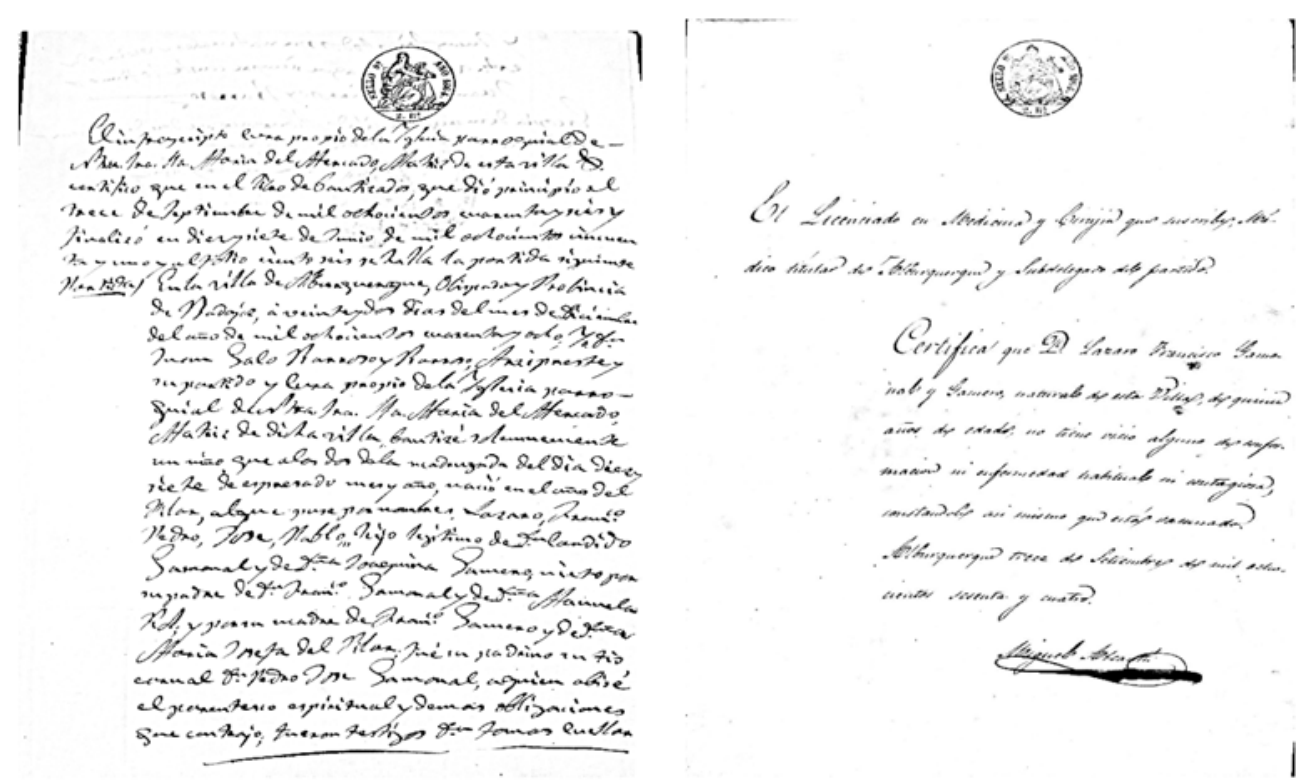

Figura 9. Partida de bautismo

Figura 10. Certificado médico
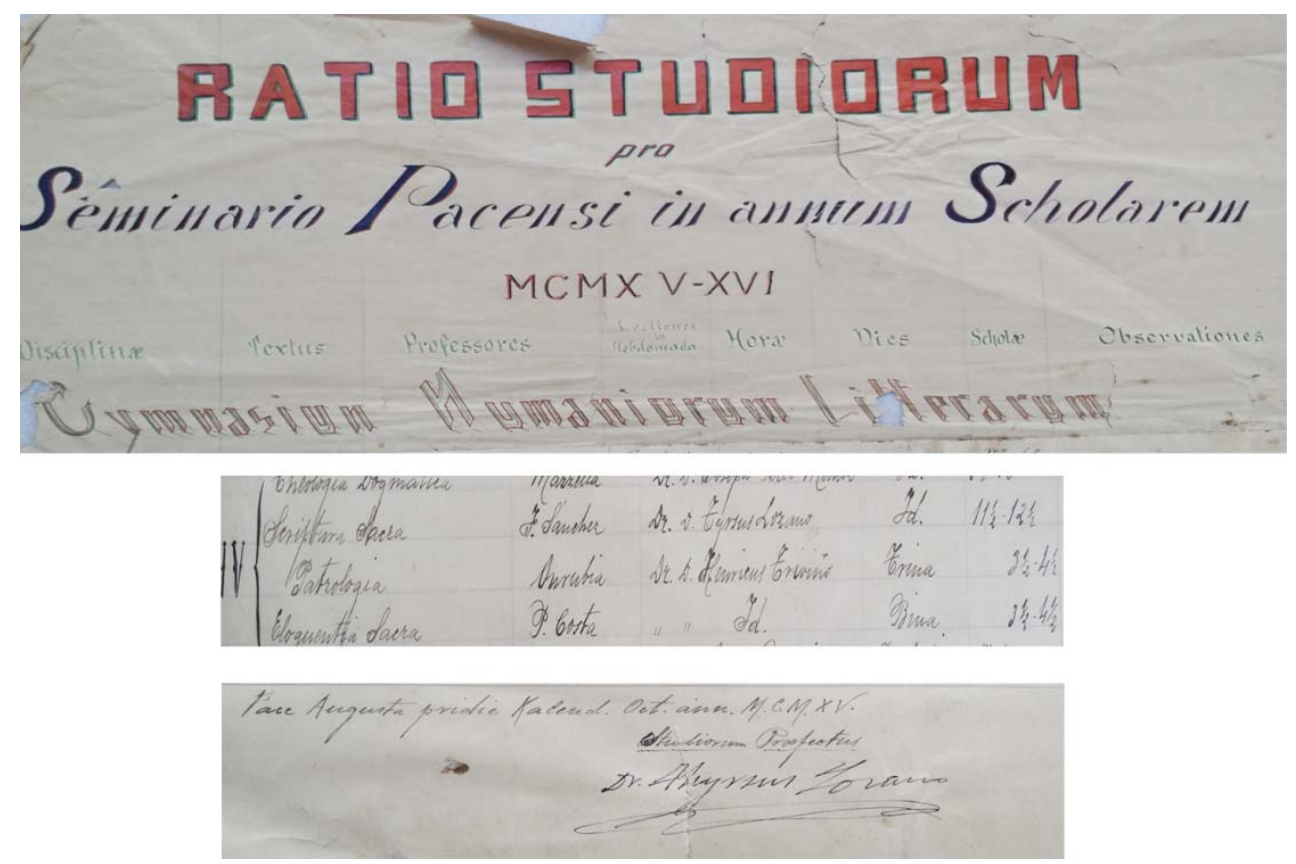

Figura 11. Plan de estudios 1915-1916 
Como pueden observar, es la serie Secretaría del cuadro de clasificación diseñado para los archivos de Seminarios la que aporta los contenidos más variados e importantes para el conocimiento de este tipo de instituciones desde el punto de la investigación en educación. Aunque no por ello es la única que debiera ser consultada para este tipo de estudios. Muy brevemente dado que no es el objeto principal de este trabajo, presentaremos otras secciones y series en las que también se localizan contenidos de carácter educativo y pedagógico.

1.0 GOBIERNO: el Concilio de Trento dejaba en manos del obispo la dirección y guía de sus seminarios. En su ayuda, colaboración y representación se encontraba la figura del rector. La dirección y gestión educativa del Seminario estaría en manos de ambos. Esta será la sección más importante que habrá de ser consultada para enmarcar cualquier investigación educativa.

1.01Documentación episcopal: para comprender qué objetivos educativos se pretendían alcanzar en los seminarios españoles es de obligado cumplimiento acudir a esta subsección. Las líneas vertebradoras de la formación ofrecida por el prelado a sus seminaristas quedarán recogidas aquí. Entre la documentación destacamos tanto el libro de fundación como las diversas constituciones y decretos ordenados para el buen funcionamiento de estos establecimientos.

1.02 Documentación rectorado: Como dijimos anteriormente, la representación legal del obispo y del seminario recae en la figura del rector, quien llevará a buen término las directrices pedagógicas y educativas designadas para el Seminario.

3.0 ADMINISTRACIÓN: La puesta en marcha y el sostenimiento de un centro requieren una adecuada administración. El investigador que desee enmarcar las circunstancias económicas que acompañaron la vida educativa de un seminario necesitará consultar esta rica documentación.

3.01 Administración general: esta subsección está constituida, por un lado, por la correspondencia generada y referida a la economía propia del Seminario; por otro, por los recibos de compras y ventas de la institución. Además podemos encontrar los recibos/justificantes de pagos emitidos por las empresas o instituciones con las que se mantuvo cualquier tipo de relación económica. Más nos interesa la serie de salarios que tiene la documentación relativa a los sueldos de los que trabajaron en el Seminario. En ella podemos corroborar la presencia de rectores, catedráticos, personal de mantenimiento y otros servicios que prestaron sus servicios al centro.

3.02 Bienes: en la documentación de esta subsección vamos a encontrar distintas series: censos, cuentas, escrituras, inventario de bienes y libro becerro. Mediante ellos se podrá comprobar cómo el sostenimiento económico de la amplia oferta educativa podía llegar sin dificultades a los más necesitados.

3.03 Obras pías: las numerosas donaciones que se hicieron con la finalidad de iniciar estos centros, además de las que se fueron fundando para tal fin, ayudan a explicar que la existencia de los mismos era una empresa de toda la Iglesia. 


\section{Conclusiones}

La Iglesia por medio de los seminarios pretendió desde mediados del siglo XVI homogeneizar la formación y aumentar el nivel intelectual del clero. Y se logrará, al menos en parte con la creación de estas nuevas instituciones que desarrollarán una importante base humanística y una especial preparación filosófica que a todas luces era insuficiente hasta el momento.

Los archivos de seminarios se convierten en testigos directos de la andadura de estos centros y son fuente única de testimonios de personas, lugares, planes de estudios, vocaciones, etc. Por todo ello, su análisis exhaustivo es hoy por hoy la única fuente fidedigna para el conocimiento preciso de estas instituciones.

Si nos centramos en la parte formativa de estas entidades, los seminarios en España fueron foco de cultura durante más de tres siglos, asumiendo el papel formativo que en muchas poblaciones españolas no existía. Por todo ello, para la realización de investigaciones sobre la historia de la educación, muy especialmente durante los siglos XVII y XVIII, se convierten en fuentes de obligada consulta. De este modo los archivos de seminarios adquieren un papel relevante dado que es en ellos donde se custodian estos contenidos. Si accedemos a los mismos nos serán de gran utilidad los cuadros de clasificación diseñados para cada entidad, muy especialmente para investigaciones educativas y pedagógicas la sección secretaria será la que nos aporte los más variados y significativos contenidos respecto a esta temática.

En el caso particular del Badajoz, podemos afirmar que el Seminario fue un centro educativo de referencia en Extremadura desde mediados del siglo XIX hasta los albores del Concilio Vaticano II. El inicio del siglo XX vio despuntar notablemente la labor educativa de San Atón comparado con otros seminarios. Confluyeron en él un excelente grupo de profesores intelectuales y humanistas que hicieron destacar al de Badajoz frente al resto de España. Un nutrido número de alumnos fueron educados en el Seminario de Badajoz. La vivencia de las virtudes fue la nota dominante del proyecto pedagógico que se estableció para ellos en cada etapa. Además, el centro posibilitó el acceso a los estudios para los que carecían de medios económicos suficientes mediante un sistema de becas. Casi la totalidad de los pueblos de la provincia, a los que sumamos poblaciones aledañas, están representados en el listado de municipios que aportaron colegiales al Seminario: ello da muestra de que realmente era un centro de referencia en Extremadura.

El archivo del Seminario custodia documentación sobre cuestiones educativas y pedagógicas muy relevantes para el conocimiento de la institución y de la historia educativa de Extremadura entre los siglos XVII-XXI. Las investigaciones educativas de carácter histórico encuentran en nuestro archivo amplia y diversa documentación respecto a alumnos, profesores, objetivos pedagógicos, planes de estudio y principios educativos. A través del cuadro de clasificación el acceso a las fuentes documentales del Seminario abre las puertas a sistemáticos conocimientos de la cultura extremeña, valorando el enriquecimiento cultural que aportó el centro a través de la educación integral que ofreció a los miles de alumnos que desde el siglo XVII han pasado por sus aulas. 


\section{Referencias bibliográficas}

AA.VV. (1992). Estudios, seminarios y pastoral en un siglo de historia de España (18821992). Roma, Colegio Pontificio Español.

Aguirre Prado, L. (1964). La Iglesia y la guerra civil. Madrid, Servicio informativo Español.

Andrés Martín, M. (1976) La supresión de las facultades de teología en las universidades españolas (1845-1855). Burgos.

Aznar, S. (1949). La Revolución Española y Las Vocaciones Eclesiásticas. Madrid, Instituto de Estudios Políticos.

Bartolomé Martínez, B. (1997). Historia de la acción educadora de la Iglesia en España. Madrid, BAC.

Cárcel Ortí, V. (1988). León XIII y Los Católicos Españoles. Pamplona.

Castro Alonso, M. (1898). Enseñanza eclesiástica en España. Valladolid, Imprenta José Manuel de la Cuesta.

Cristiani, L. (1977). El Concilio de Trento. (1518-1534). En Fliche y Martin (dir.): Historia de la Iglesia. Valencia, Edicep.

Cuenca Toribio, J. M. (1973). Historia de España. Barcelona.

Cuenca Toribio, J.M. (1973). Notas para el estudio de los seminarios españoles en el pontificado de Pío IX, Saitabi, 23, 51-88.

Faubell Zapata, V. (1993). Autonomía y universidades de la Iglesia en España, Revista de ciencias de la educación, 154, 213-219.

Fernández Conde, M. (1948). El decreto tridentino sobre seminarios y su aplicación en España hasta el año 1723. Madrid.

Fernández Conde, M. (1948). España y los seminarios tridentinos. Madrid, Consejo Superior de Investigaciones Científicas.

Fuentes Nogales, M.C. y Pérez Ortiz, G. (2014). Guía del Archivo del Seminario Metropolitano San Atón. Badajoz, Seminario Metropolitano san Atón.

Infantes Florido, J.A. (1977). Un Seminario de su siglo: entre la Inquisición y las Luces. Las Palmas.

Martín Hernández, F. (1979). La formación del clero en los siglos XVII y XVIII. En García Villoslada, R. (coord.): Historia de la Iglesia en España. Madrid, BAC.

Martín Hernández, F. y Martín Hernández, J. (1973). Los seminarios españoles en la época de la Ilustración: Ensayo de una pedagogía eclesiástica en el siglo XVIII. Madrid, Consejo Superior de Investigaciones Científicas.

Martín Hernández, F. (1973). Los seminarios españoles en la época de la Ilustración: Ensayo de una pedagogía eclesiástica en el siglo XVIII. Madrid, Instituto Enrique Florez.

Martín Hernández, F. (1964). Los seminarios españoles: Historia y pedagogía. Salamanca, Sígueme.

Martín Velasco, J.D. (1996). Avatares del clero español en los últimos años. Santander, Sal Terrae.

Orlandis, J. Informe de la Visita apostólica a los seminarios españoles en 1933-1934 (2007). Edición del Informe y estudio sobre "La formación sacerdotal en España" (1850-1939), Anuario Historia de la Iglesia, 16, 517-518.

Rops, D.(1970). Historia de la Iglesia. Madrid.

Rubio Merino, P. (1964). El Seminario de San Atón. 1664-1964. Madrid, Artes gráficas Maribel.

Sánchez Aliseda, C. (1942). La doctrina de la Iglesia sobre los Seminarios desde Trento hasta nuestros días. Granada, Facultad de Teología.

Tineo, P. (1993). La formación teológica en los seminarios españoles (1890-1925), Anuario de Historia de la Iglesia, 2, 45-96. 
Turín, I. (1967). La educación y la escuela en España de 1874 a 1902. Madrid. Vázquez Vilanova, J.A. (2002). Los estudios en los seminarios españoles en el siglo XIX, Hispania Sacra, 54, 109, 227-242.

Vergara Ciordia, J. (1996). Mentalidad cristiana y pensamiento pedagógico de la Iglesia en España. En Martínez, B. (coord.): Historia de la acción educadora de la Iglesia en España. Madrid, BAC.

Vergara Ciordia, J. (2004). Historia y pedagogía del Seminario Conciliar en Hispanoamérica (1563-1800). Madrid, Dykinson.

Vergara Ciordia, J.; Comellas Gutiérrez, B. (2014). El seminario conciliar en las relaciones Iglesia-Estado en España desde Trento al Concilio Vaticano II, Revista de Estudios Extremeños, 70/n $\mathrm{n}^{\mathrm{O}}$ extraordinario, 553-596.

Vergara Ciordia, J.; Rodríguez Sedano, A. (2014). Devenir institucional de la formación sacerdotal hasta el Concilio de Trento, Revista de Estudios Extremeño,s 70/n ${ }^{\circ}$ extraordinario, 511-552. 